University of Nebraska - Lincoln

DigitalCommons@University of Nebraska - Lincoln

2011

\title{
The radiation quality factor as an Ornstein-Uhlenbeck process
}

\author{
A. F. Barghouty \\ NASA Marshall Space Flight Center, Huntsville, AL
}

Follow this and additional works at: https://digitalcommons.unl.edu/nasapub

Part of the Physical Sciences and Mathematics Commons

Barghouty, A. F., "The radiation quality factor as an Ornstein-Uhlenbeck process" (2011). NASA

Publications. 44.

https://digitalcommons.unl.edu/nasapub/44

This Article is brought to you for free and open access by the National Aeronautics and Space Administration at DigitalCommons@University of Nebraska - Lincoln. It has been accepted for inclusion in NASA Publications by an authorized administrator of DigitalCommons@University of Nebraska - Lincoln. 


\title{
The radiation quality factor as an Ornstein-Uhlenbeck process
}

\author{
A.F. Barghouty* \\ Space Science Oce, NASA Marshall Space Flight Center, Huntsville, AL 35812, USA
}

\section{A R T I C L E I N F O}

\section{Article history:}

Received 18 May 2010

Accepted 30 November 2010

\section{Keywords:}

Space radiation

Radiation quality factor

Monte-Carlo simulations

Stochastic processes

Ornstein-Uhlenbeck process

\begin{abstract}
A B S T R A C T
Radiation protection strategies for extended or deep space missions rely on accurate and robust estimates of exposure dose and corresponding risk to crew health, systems functions, and mission safety in general. Simulation and modeling of dose and risk associated with such exposures are, to various degrees, made difficult by the inherent complexity and variability in characterizing the radiation environment, its passage and interaction with matter, and its biological effects. One of the more important contributors to the overall uncertainty in dose or risk assessment is the empirical variability in the radiation quality factor, $Q$, which is typically used to differentiate such effects. Motivated in part by recent Monte-Carlo based simulations of this variability, we propose and demonstrate a stochastic dynamic model for $Q$ based on the Ornstein-Uhlenbeck process. The proposed model's probability density function is a Gaussian in $Q$ and with a linearly (in the logarithm of the LET variable) growing variance, but with rather complex scaling properties. The model's density function is shown to be quite sensitive to any functional parameterization of the mean (deterministic) behavior of the quality factor with a discontinuous first derivative in LET. The proposed linear stochastic model is also shown to be insensitive, however, to the precise functional form of the deterministic $Q$.
\end{abstract}

Published by Elsevier Ltd.

\section{Introduction}

In addition to other flight risks and hazards, space flights beyond the confines and protection of the Earth's atmosphere and magnetic field also face the challenges of space radiation exposure. In extended lunar surface missions, for example, protection of crew and critical systems require shielding strategies against a host of radiation fields, both natural and man-introduced. In future manned, nuclear-powered space treks to Mars, special shielding requirements are unavoidable. Due to various degrees of variability, unpredictability, as well as - in some critical areas - lack of basic data, guaranteeing safe levels of exposure poses a special challenge. Exposure estimates for shielding solutions as well as for safety assessment must be formulated and optimized based on incomplete data, constrained by both technical and non-technical factors. One of the more consequential constraints, albeit somewhat subjective, is that of ALARA, "as low as reasonably achievable."

To various degrees, such estimates are hampered by uncertainties; in our basic knowledge of the radiation environment itself, its transport and interaction in various media of complex geometry and composition, and most critically, in the human biological response to such exposures. The empirical variability in the so-

\footnotetext{
* Tel.: +1 256961 7508; fax: +1 2569617522.

E-mail address: abdulnasser.f.barghouty@nasa.gov.
}

called radiation quality factor, which is used to differentiate among the various radio-biological effects and responses, is now known to be a major contributor to the overall variability. On the one hand, experimental and theoretical studies, both physical and biological, can in principle reduce these uncertainties. On the other, computational and statistical studies can do their part in either narrowing and/or rigorously bounding risk estimates in the presence of these uncertainties.

In some applications, Monte-Carlo based analyses are known to suffer drawbacks (Ferson, 1996) in estimating exceedance risks (the frequency or probability that an uncertain quantity exceeds a certain threshold or limit). In risk analysis studies in particular, Monte-Carlo based simulations may be unable to propagate non-statistical uncertainty or to bound the risk estimates. Given the empirical nature of biological uncertainties, one is forced to perform probabilistic uncertainty analysis of probabilistic estimates. This approach can severely overestimate or underestimate the risk estimates (Hoffman and Hammonds, 1994; Ferson and Tucker, 2006).

An alternate approach based on probability theory and interval analysis is described in Ferson and Tucker (2006). Another, is the use of fuzzy logic and fuzzy arithmetic in uncertainty analysis where a fuzzy number is considered a generalization of an interval (Ferson, 1992; Rajkumar and Johnson, 2001). In this notion, a fuzzy number is a stack of infinitely many intervals each of which represents an estimate of confidence about the uncertainty. 
While some or all of these approaches may well be applicable in addressing and modeling the uncertainty in the radiation quality factor, only Monte-Carlo type analyses have thus far been used in a surprisingly limited number of studies focusing on this critical issue. The recent Monte-Carlo study reported by Cucinotta et al. (2005) shows remarkably large uncertainties in the estimated dose and risk estimates due, largely, to the uncertainty (variability) in the radiation quality factor. For shielding purposes, for example, the presence of these large uncertainties can frustrate any and all potential solutions. Studies to reduce these uncertainties, both in simulations as well as empirically, are clearly needed.

Our approach is to model the radiation quality factor as a stochastic dynamical process in order to better understand these fluctuations through closer characterization - and thus sensitivity - to model parameters. This approach is motivated in part by some simulated characteristics of the quality factor (done by the aforementioned study (Cucinotta et al., 2005)) which seem to suggest the Ornstein-Uhlenbeck process as a possible stochastic dynamic model for it.

This paper is organized as follows. A brief description of the relevant radiation environment and exposure doses are given first in Section 2. This is followed in Section 3 by a recap of the MonteCarlo simulations of the quality factor. We propose and analyze in Section 4 the time-dependent Ornstein-Uhlenbeck process as a stochastic model for a fluctuating quality factor, including detailed analysis of sensitivities to both parameters and formulations. Section 5 offers a summary and some suggestions for future directions.

\section{The space radiation environment}

Energetic, high-charge galactic cosmic-ray ions (GCR) and solar energetic particles (SEP) constitute the main (natural) source of this intense radiation environment. The energy range of these particles spans more than eight orders of magnitude (from thermal to ultrarelativistic) while their atomic numbers populate the entire stable nuclides of the periodic table.

Atomic charges of 1 (hydrogen) though 26 (iron), however, are considered important for radiation safety and shielding purposes. By number, hydrogen constitutes about $90 \%$, helium $7 \%$, and all others $3 \%$ of the GCR ions. The intensity of the ambient GCR component $\left(\sim 1 \mathrm{~cm}^{-2}\right)$ peaks around $500 \mathrm{MeV} /$ nucleon and is modulated by a factor of about three over the 11-year solar cycle (e.g., Kudela et al., 2000). During solar maximum and due to the actions of the solar wind, access to the heliosphere by diffusing GCR ions is reduced. As a result the GCR component appears depressed in the inner heliosphere.

During heightened solar activities, solar particle events, while random in occurrence, are more frequent and strong enough to transport SEPs (by a propagating shock driven by a coronal mass ejection or CME) to Earth's orbit and beyond. The SEP component is mostly composed of energetic protons, peaks around few tens of $\mathrm{MeV}$ in energy, but can vary widely in intensity $\left(\sim 10^{7} \mathrm{~cm}^{-2}\right)$ as well as in the shape of its energy spectra. The so-called 'large' events, e.g., the October 1989-event, can be an order of magnitude more intense than the 'average' event, and many orders of magnitude above the quiescent conditions, lasting hours to 2-3 days (e.g., Kudela et al., 2000).

In addition to these natural sources of space energetic particles, there likely to be man-introduced radioactive and fission sources for power and propulsion purposes as well. A number of studies (Kerslake, 2005; Davis et al., 2005) for the power requirements during future lunar surface missions, for example, suggest that the need is on the order of tens of kilo-watts of electric power. For this level of power chemical, solar, and radio-isotope sources may be insufficient or impractical. For radiation protection purposes, fission reactors are considered mainly as sources of energetic neutrons and gamma rays (photons). Contributions of these sources to the total expected dose (Barghouty, 2007) are due mostly to prompt neutrons. Most of these are energetic or 'fast' neutrons produced (at $\sim 10^{14} \mathrm{~cm}^{-2}$ ) as direct fission products with an average energy of about $2 \mathrm{MeV}$. Neutrons can also be produced as spallation products of GCR and SEP interactions with either lunar surface or shielding materials. Adams et al. (2007) focused on the contribution of this neutron albedo to the total dose and found it to be, for some special cases, non-negligible. Photons (at $\sim 10^{10} \mathrm{~cm}^{-2}$ ) are produced both as direct products of the fission reaction as well as a result of the subsequent decay of the fission radioactive products. For shielding purposes, however, gamma rays with energy less than 0.6 MeV are typically ignored (e.g., Angelo and Buden, 1985).

\subsection{Expected exposure levels}

When these particles traverse a medium (e.g., a tissue or a shielding material) they undergo both atomic and nuclear collisions with the medium's nuclei, atoms, and electrons. These collisions deposit energy and produce secondary components, like neutrons, in addition to leading to the fragmentation of both GCR and target nuclei. It is these nuclear and atomic collisions, their resulting tracks and cascading structure in the medium along with their frequency and severity that result in their special energy deposition characteristics (e.g., Wilson et al., 1991) that make the GCR and SEP components the health hazards they are. It is estimated that in a 2-year Mars excursion, for example, as many as half of all the cells of an astronaut's body will be traversed by GCR ions.

Two key (physical) components in dose and risk assessment models are the radiation environment and the radiation fields transport and interactions in the medium. With these, the absorbed dose (energy per unit mass, in Gy) can be estimated rather well. However, since different radiations are known to produce different biological effects for the same delivered dose, a quantity that is more indicative of the expected health risk is the so-called doseequivalent (in Sv). Dose-equivalent is calculated from the (physical) dose corrected by a dimensionless, multiplicative factor called the radiation 'quality factor', or simply, the $Q$-factor. Ionizing radiation like energetic heavy ions (e.g., GCR ions) is characterized by high $Q$ values. Uncharged neutrons are also assigned high $Q$ values to underscore their more serious health hazards relative to either $\mathrm{X}$-rays or gamma rays at the same energy.

Current National Commission on Radiation Protection and Measurement (NCRP) dose limits for a one-year exposure is $0.5 \mathrm{~Sv}$ (for bone morrow in low Earth orbit) (NCRP, 1999). Outside the magnetosphere, GCR during solar minimum are estimated to contribute about this much alone per year, while a large solar particle event, like the August-1972 one, can impart up to 1-2 Sv. On the lunar surface, the dose due to GCR and SEP is expected to be reduced by a half due to the shadow shielding effect of the Moon itself. The introduction of a small nuclear fission reactor $(\sim 25-\mathrm{kW})$ is estimated to add about $5 \mathrm{cSv}$ per year at a safe distance from its shielded core. Ignoring this contribution, the expected (Barghouty and Thibeault, 2006) GCR and SEP annual dose with and without a 50-cm shield made of idealized lunar regolith are 20 and $50 \mathrm{cSv}$, respectively. Given currently accepted limits, extended $(\gtrsim 6$ months) crewed surface missions will clearly require shielding solutions (e.g., Wilson et al., 1997).

Biological effects of the passage of energetic and charged particles (e.g., GCR or SEP ions) through cells and tissues are poorly known in addition to being difficult to study. This is in large part due to limited exposure data. As a consequence, estimating the health risks associated with space radiation exposure is hampered 
mostly by uncertainties in the biological response to such particles (e.g., Cucinotta et al., 2005). Other factors associated with the radiation environment, its physical interactions, as well as with dose and dose-rate volatilities, also contribute.

\section{Monte-Carlo modeling of the $Q$-factor}

Unlike the physically describable and measurable dose, the $Q$ factor is a dimensionless proxy variable assumed to 'represent' the majority of the biological effects associated with exposure to ionizing radiation - but without specifying such effects by their end points or response functions. As such, the $Q$-factor is not the same as an 'index' for an average relative biological effectiveness (RBE) of the radiation at a given dose (e.g., Alpen, 1998). Rather, it is a consensus value agreed upon to quantify the relative health effects of one type of radiation exposure to another (ICRP, 2003). In this respect, the $Q$-factor is a useful and convenient measure of the exposure dose and its expected health effects. Lack of sufficient experimental data, however, coupled with the large number of pathways to the biological effects of radiation exposure inevitably produce large uncertainties in 'assigning' $Q$-factor values.

The $Q$-factor is taken to be dependent on the linear energy transfer (LET), $L$ in units of energy per length, of the radiation field. This dependence - while complex and highly variable - is typically modeled as a one-to-one mapping, $Q: \mathbb{R}_{+} \rightarrow \mathbb{R}_{+}$. Energetic charged particles of space radiation (as well as their interaction products, most notably neutrons) are characterized as high-LET particles. Variations in the quality as well as quantity of high-LET exposure data, in addition to other difficult-to-quantify factors, further inflate the uncertainties in the Q-factor (ICRP, 2003).

Based on track structure models (e.g., Katz et al., 1971), and over a rather wide LET range, $Q(L)$ is treated as a stochastic variable in $L$ but with persistent average behaviors for low, intermediate, and high values of $L$. On average, for low values of $L, Q$ is seen to vary little with $L$. For intermediate values of $L$ and up to some characteristic value, $L_{m}, Q$ tends to increase rapidly with $L$. Whereas for values higher than $L_{m}, Q$ begins to decrease, but less rapidly, with increasing $L$.

This average dependence of $Q$ on $L$ is heuristically depicted as a piecewise continuous function of $L$ as:

$Q(L)= \begin{cases}1 & L_{0}>L \\ a L-b & L_{m}>L \geq L_{0} \\ c L^{-p} & L \geq L_{m}\end{cases}$

In this particular depiction, the parameters $L_{0}, L_{m}$, and $p$ are taken to be stochastic independent variables where $L_{m}$ represents the maximum LET that yields the greatest $Q$ value, $Q_{m}$. The fit parameters $a, b$, and $c$ are calculated using the above functional form along with the sampled values for $L_{0}, L_{m}, p$, and $Q_{m}$, taken, independently, from their respective empirical distributions (Cucinotta et al., 2005). Using this form, the ICRP-60 recommended $Q$-factor (ICRP, 1991) is characterized by $L_{0}=10 \mathrm{keV} / \mu \mathrm{m}, L_{m}=100 \mathrm{keV} / \mu \mathrm{m}$, $p=1 / 2$, and $Q_{m}=30$. These values fix the parameter $a$ at $0.32, b$ at 2.2 , and $c$ at 300 (Cucinotta et al., 2005). Similarly, the ICRP-26 recommended $Q$-factor (ICRP, 1977) corresponds to $L_{0}=3.5 \mathrm{keV} /$ $\mu \mathrm{m}, L_{m}=172.5 \mathrm{keV} / \mu \mathrm{m}, p=0$, and $Q_{m}=20$, resulting in $a=0.11$, $b=-0.06$, and $c=20$.

Sensitivity analysis of $Q$ to its parameters (Aziz, 2007) as given by Eq. (1) suggest that $L_{0}$ and $p$ have minor effects on the uncertainty in $Q$; except for either exceedingly low or exceedingly highLET values. Low LET values contribute little to the variability in $Q$ whereas high-LET values contribute little to the total dose. In contrast, in the intermediate LET region $(\sim 100 \mathrm{keV} / \mu \mathrm{m})$ variation in $L_{m}$ produces significant variability in $Q$ e.g., variability in the location of the 'cusp' point $\left(L_{m}, Q_{m}\right)$. Typical Monte-Carlo based simulations assume these 'structure' variables to be independent. Lack of characteristic data on how these variables affect each other, both empirically as well as in simulations, will always produce large variations in $Q$, irrespective of the assumed distributions.

On the one hand, the recent Monte-Carlo based simulations of the $Q$-factor (Cucinotta et al., 2005) using Eq. (1) with empirically suggested distributions for $L_{0}, L_{m}, p$, and $Q_{m}$ appear to give large ( $\gtrsim 200 \%$ ) LET-dependent uncertainties in the estimated dose and risk estimates. On the other, and for purely modeling purposes like ours here, the study also seems to suggest two interesting and consequential features about the simulated variability and average behavior of the $Q$-factor: (1) the simulated noise appears to be additive (rather than multiplicative) as evident by the remarkably stable ratio of the simulated standard deviation to the simulated mean of the $Q$-factor (Fig. 1), and (2) the simulated mean appears to have a persistent functional form or structure (Fig. 1). In what follows we shall use these two salient features to propose a stochastic dynamical model for the $Q$-factor based on a timedependent Ornstein-Uhlenbeck stochastic process.

\section{The $Q$-factor as a linear stochastic dynamical system}

\subsection{The proposed linear model}

In analogy of deterministic dynamical systems being governed by ordinary or partial differential equations, stochastic dynamical systems are governed by stochastic differential equations (SDE) of the form (see e.g., Gardiner, 1983; Lefebvre, 2000):

$d x=C(x, t) d t+\sqrt{D(x, t)} d W$,

where for the scalar random variable $x(t), C(x, t)$ is its drift (or mean) term and $D(x, t)$ is its diffusive (or volatile) term, and $d W$ is a Wiener process (i.e., a Gaussian white noise). For a linear SDE, $C$ is a linear (deterministic) function of $x$ while $D$ is another deterministic function but independent of $x$. A time-dependent Ornstein-Uhlenbeck (OU) process -a well-studied linear process- has the simple form:
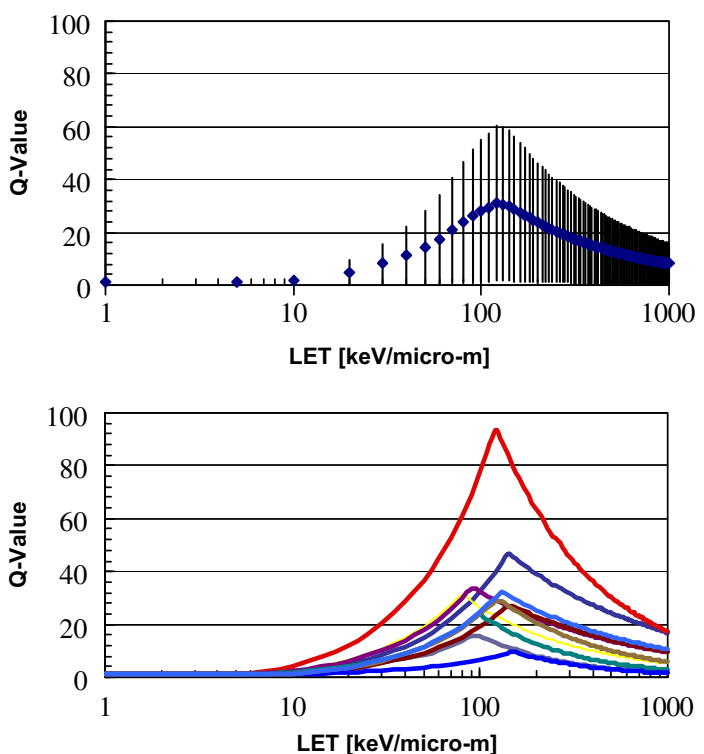

Fig. 1. The additive noise and persistent average structure in the Monte-Carlo simulated $Q$-factor: Error bars on the top part are one standard deviations while the data points depict the mean as a function of LET. The bottom part shows a number of realizations for a variable $Q$ (From Aziz (2007) based on the formulation in Cucinotta et al. (2005)). 
$d x=C(t) x d t+\sqrt{D(t)} d W$.

With time $t$ replaced by the logarithm of $L$, i.e., $t \leftrightarrow \ell=\log _{10} L$, the proposed OU-based SDE for $Q(\ell)$ is then,

$d Q=C(\ell) Q d \ell+\sqrt{D(\ell)} d W$.

Next, we need to estimate the drift and diffusion functions for this equation. The drift term is assumed to have such a form so as to reproduce the average behavior of $Q(\ell)$ in the limit when $D \rightarrow 0$, e.g., the one depicted in Eq. (1). For the OU process, this is simply,

$C(\ell)=\frac{1}{\langle Q\rangle} \frac{d\langle Q\rangle}{d \ell}$,

independent of the exact form of the mean, $\langle Q(\ell)\rangle$, but which is assumed to depend explicitly on $\ell$. We require, however, $\langle Q(0)\rangle$ to be fixed (i.e., deterministic) and $\langle Q(\ell>0)\rangle$ to be positive definite.

Since we are interested in the solutions of Eq. (4) over a continuous and finite domain of $\ell$, say $\ell \in[0,4]$, we require a continuous $\langle Q(\ell)\rangle$. To that end, we have fitted the mean, MonteCarlo simulated $Q$ (see Fig. 1 ) with the following variance-gamma function (distribution):

$\langle Q(\ell)\rangle=Q_{s} \frac{\left(\alpha^{2}-\beta^{2}\right)^{\lambda}}{\Gamma(\lambda)(2 \alpha)^{\lambda-1 / 2}}|\ell-\mu|^{\lambda-1 / 2} K_{\lambda-1 / 2}(\alpha|\ell-\mu|) \exp [\beta(x-\mu)]$.

In the above, $Q_{S}$ is a constant, $\Gamma$ is Euler's gamma function, $K_{\lambda-1 / 2}$ is the modified Bessel function of the second kind with fractional order, and $\alpha, \beta, \lambda$, and $\mu$ are fixed parameters. A best fit to the MonteCarlo simulated mean of $Q$ (see Fig. 2) yielded the following values for the fit parameters: $Q_{s}=33, \alpha=1.84, \beta=0.4, \lambda=1.0$, and $\mu=2.09$. The choice for the variance-gamma function in particular is due to its explicit parameterization of (1) the thickness of the tail $(\alpha),(2)$ the asymmetry $(\beta)$ around the peak value, (3) the overall scale $(\lambda)$, and (4) the location of the peak $(\mu)$, all characterizing the mean behavior of $Q$. This is in addition to our desire to preserve the 'cusp' like structure in this mean behavior of $Q$. This is manifest (like in Eq. (1)) in a discontinuous first derivative of $\langle Q(\ell)\rangle$ at the peak. The reason for preserving this feature will be expounded upon in Section 4.3.

For the diffusion term, and based on the Monte-Carlo simulations of Cucinotta et al. (2005), Section 3, which seem to suggest that the noise in the simulated $Q$-factor is an additive noise, $D(\ell)$ can be simply written as:

$D(\ell) \approx \frac{1}{2}(\langle Q\rangle)^{2}$.

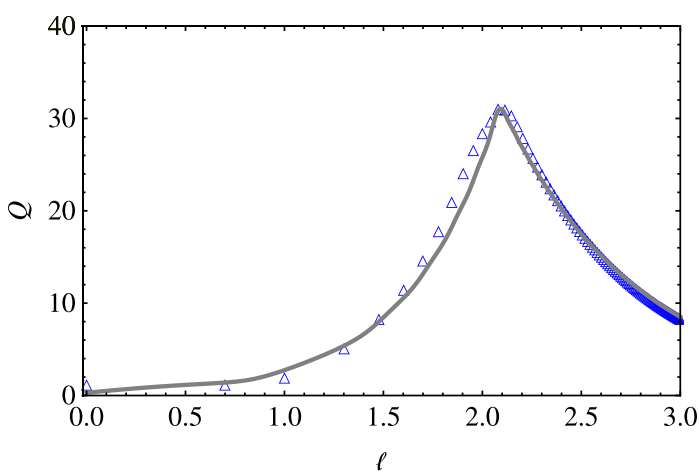

Fig. 2. A fit (solid line) to $\langle Q(\ell)\rangle$ according to Eq. (6). Discrete data points are the Monte-Carlo simulated mean values of the $Q$-factor as depicted in Fig. 1.
The stochastic process as described above, which we propose as a model for the variability and mean behavior of the $Q$-factor, is that of a time-dependent Ornstein-Uhlenbeck process with the normalized LET variable, $\ell$, playing the role of the continuous time variable.

\subsection{Solution of the linear system}

Mathematically, the OU process is a prototypical stochastic process with simple and explicit solutions to both its stochastic differential equation, Eq. (4), as well as its corresponding Fokker-Planck equation (Gardiner, 1983):

$\frac{\partial f_{Q}(Q, \ell)}{\partial \ell}=-\frac{\partial}{\partial Q}\left[C(\ell) Q f_{Q}(Q, \ell)\right]+\frac{1}{2} D(\ell) \frac{\partial^{2}}{\partial Q^{2}}\left[f_{Q}(Q, \ell)\right]$,

where $f_{Q}(Q, \ell)$ is the probability density function (PDF) for $Q$. Moreover, the Ornstein-Uhlenbeck process is characterized by a bounded variance and a stationary probability density function. The process is typically used to model noise in a system characterized by noise correlations that do not depend sensitively on the exact form or structure of the correlation function. Physically, the OU process idealizes Brownian motion in a harmonic potential with reflecting boundaries.

Lo and Hui (2006) derived a general solution to Eq. (8) for a time-dependent OU process. For a known (deterministic) value for $Q$ at $\ell=0, Q_{0}$, the solution is:

$f_{Q}\left(Q, \ell ; Q_{0}, 0\right)=\frac{1}{\sqrt{4 \pi q_{1}(\ell)}} \exp \left\{-\frac{\left[Q \exp \left(q_{2}(\ell)\right)-Q_{0}\right]^{2}}{4 q_{1}(\ell)}+q_{2}(\ell)\right\}$,

where,

$q_{1}(\ell)=(\langle Q(0)\rangle)^{2} \ell$,
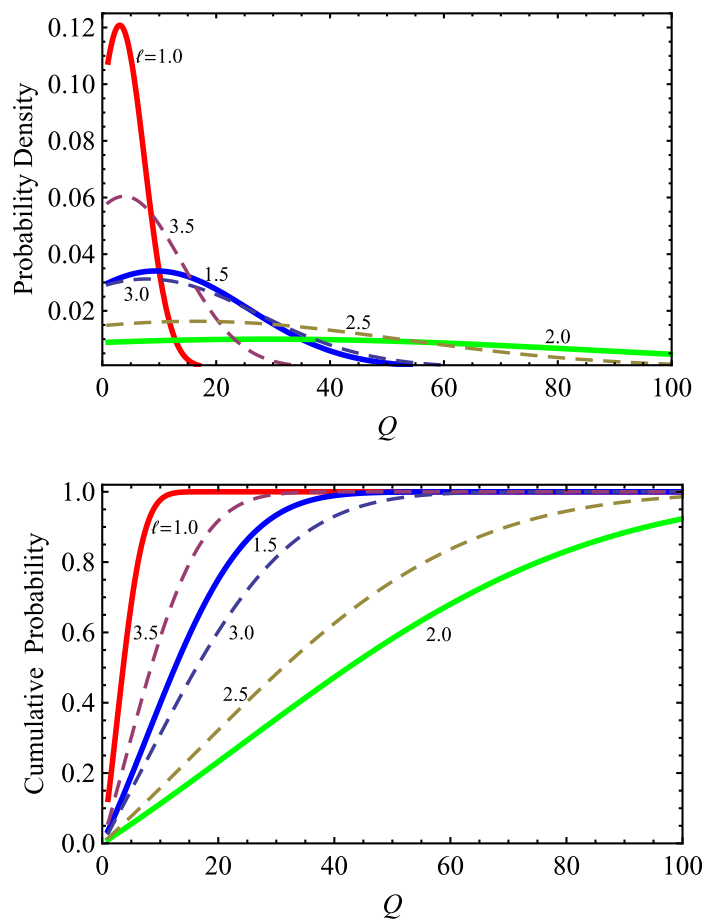

Fig. 3. The OU-based probability density function (top) and cumulative distribution function (bottom) of the $Q$-factor based on Eq. (9) for various values of $\ell .\langle Q(\ell)\rangle$ is calculated according to Eq. (6) 


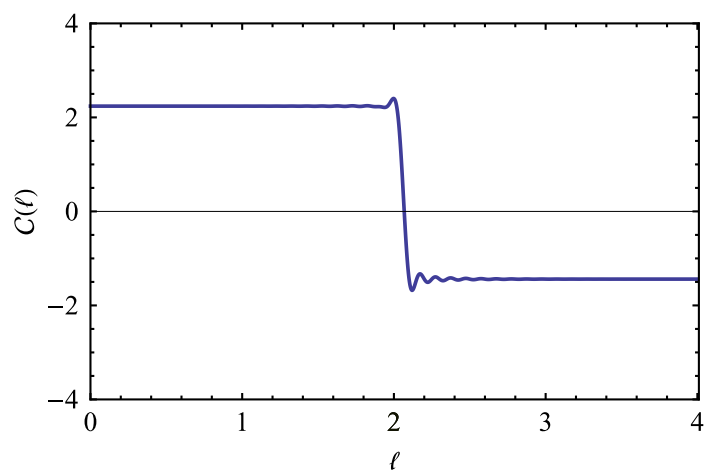

Fig. 4. Discontinuity in the drift term, Eq. (5), due to the discontinuity in the first derivative of $\langle Q(\ell)\rangle$, Eq. (6).

and,

$q_{2}(\ell)=\ln \left(\frac{\langle Q(0)\rangle}{\langle Q(\ell)\rangle}\right)$

\subsection{Analysis - parametric sensitivity}

Eq. (9) along with the supporting functions and parameters as described in the previous section can now be readily used to calculate the moments of $Q$. Note that the PDF for $Q$ also depends on ८. Fig. 3 depicts the behavior of this OU-based PDF for $Q$ for different values of $\ell$. The PDF, normalized to unity over the range of $Q \in$ $[0.1,1000]$ for each $\ell$, is an evolving (in $\ell$ ) Gaussian of $Q$. Fig. 3 also shows the corresponding cumulative distribution function (CDF) for $Q$ at the same values of $\ell$.

The spread in this Gaussian PDF is not monotonic, however, even though the variance is a monotonically increasing function of $\ell$, Eq. (10). For when $\ell$ approaches $\mu$ (with has a fitted value of 2.09 for our assumed $\langle Q(\ell)\rangle$, Eq. (6)), the spread is reversed and the PDF actually begins to shrink. This behavior of the PDF is a direct consequence of the first derivative of $\langle Q(\ell)\rangle$ being discontinuous at the peak (i.e., at $\ell=\mu$ ). This makes the drift term also discontinuous at the peak, Eq. (5) and Fig. 4. As a result, $f_{Q}(Q, \ell)$ asymptotically approaches zero for $\ell<\mu$ and $\delta\left(Q-Q_{0}\right)$ for $\ell>\mu$, due to the presence of the discontinuity in $C(\ell)$ at $\ell=\mu$.

This solution behavior will be characteristic of any assumed form of $\langle Q(\ell)\rangle$ with a discontinuous first derivative at the peak, like Eq. (6), (Fig. 4) or Eq. (1), (Fig. 5). Fig. 6 shows the OU-based PDF and CDF functions, given Eq. (1) for the mean behavior of $Q$. The same behavior is seen for Eq. (1) as it is for Eq. (6). However, when the

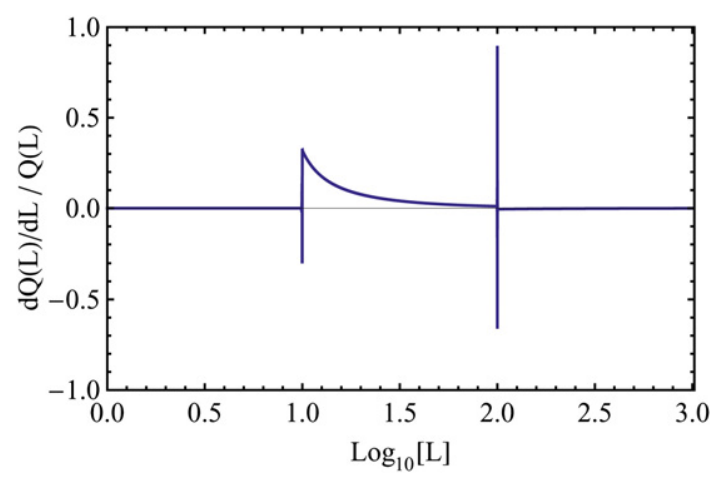

Fig. 5. Discontinuity in the first derivative of $Q(L)$ w.r.t. $L$ according to Eq. (1).
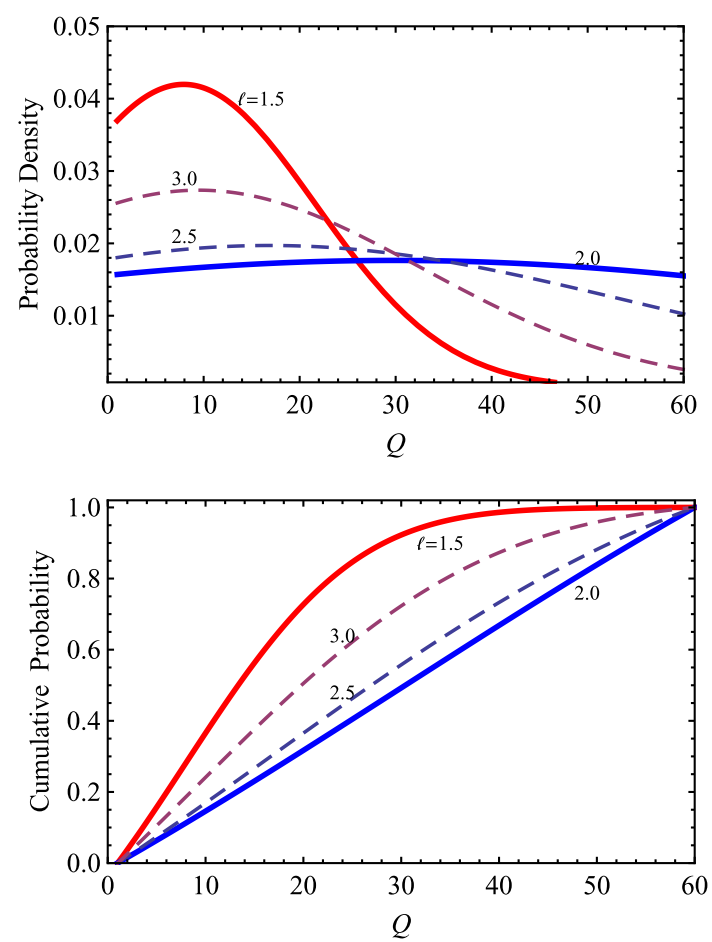

Fig. 6. The OU-based probability density function and cumulative distribution function of the $Q$-factor based on Eq. (9) with $\langle Q(\ell)\rangle$ calculated according to Eq. (1).

discontinuity in the assumed form of the mean of $Q$ is smoothed out, e.g., for $\lambda>1.4$ for Eq. (6), a spreading Gaussian that approaches zero asymptotically is easily recovered, as can be seen in Fig. 7 .

Fig. 8 depicts the mean behavior of $Q$ along with its standard deviations as functions of $\ell$, using the default parameter set: $\{\alpha=1.84, \beta=0.4, \lambda=1.0, \mu=2.09\}$ in calculating $\langle Q(\ell)\rangle$ according to Eq. (6). Fig. 9 shows the same when $\langle Q(\ell)\rangle$ is calculated according to Eq. (1).

While it is straightforward to model the deterministic or mean behavior of $Q$ as well as the additive noise, separately, a machinery like the OU process (a time-dependent OU process in this particular case) is required to capture both and consistently, which what Fig. 9 shows when compared to the Monte-Carlo simulated results in Fig. 1.

We focus next on the sensitivity of the OU-based PDF to the model's structure parameters $(\alpha, \beta, \lambda$ and $\mu)$ of Eq. (6) which characterize both the mean and volatile behaviors of $Q$ through the drift, $C(\ell)$, and diffusion, $D(\ell)$, deterministic functions. Fig. 10 shows plots of the first partial derivatives of the PDF to the structure

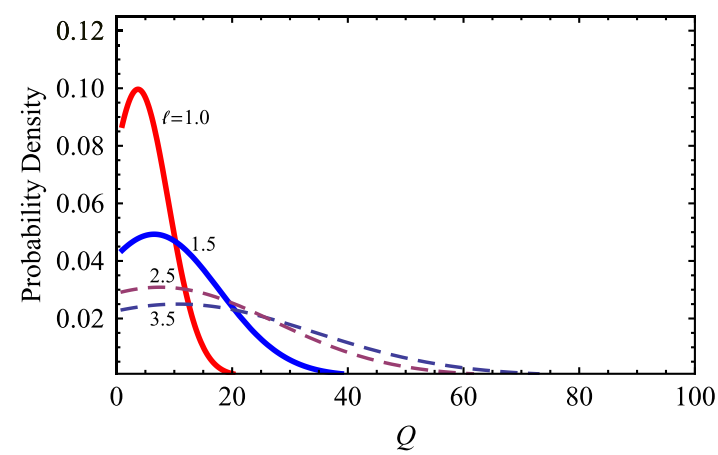

Fig. 7. The OU-based probability density function of the $Q$-factor, similar to that on Fig. 3, but for a scale parameter $\lambda=3.0$ instead of the default value of 1.0 


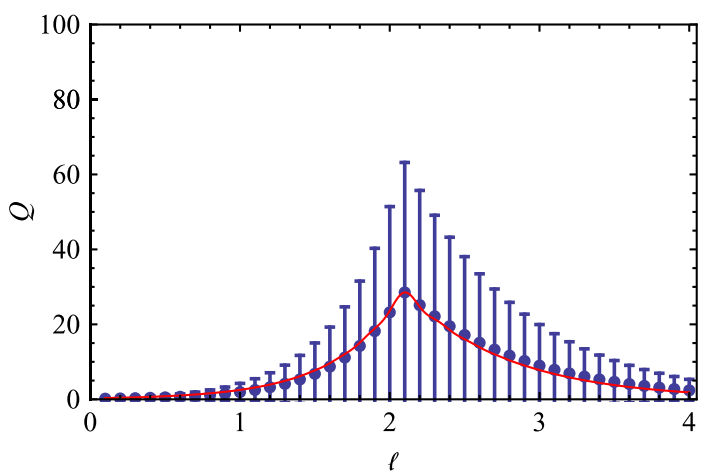

Fig. 8. The calculated mean $Q$ (circles) along with its standard deviation according to the OU-based PDF, Eq. (9). Solid line is the deterministic $Q$ as given by Eq. (6).

parameters - holding the rest fixed at their respective default values - calculated at a fixed $Q$ and $\ell$. For the asymmetry parameter $\beta$, for example, this is defined as,

$S_{\beta}(\beta) \equiv \frac{\partial f_{Q}}{\partial \beta} /\left.f_{Q}\right|_{\left\{Q^{\star}, l^{\star}, \alpha^{\star}, \mu^{\star}\right\}}$,

where the set $\left\{Q^{*}, \ell^{*}, \alpha^{*}, \mu^{*}\right\}$ refers to the default values for the parameters as described above and $Q^{*}=\left\langle Q\left(\ell^{*}=2\right)\right\rangle$. Functions $S_{\alpha}$, $S_{\lambda}$ and $S_{\mu}$ are defined similarly. The rather smooth behaviors of $S_{\alpha}(\alpha>\beta)$ and that of $S_{\alpha}(\alpha<\beta)$ as depicted in Fig. 10 suggest that the OU-based PDF is rather insensitive to the tail structure's or the asymmetry's exact parameterization.

It is, on the hand, quite sensitive to the scale parameter $\lambda$, as has been alluded to earlier. The fitted mean behavior of $Q$ is consistent with a $\lambda=1.0$, which is close to the zero of the $S_{\lambda}$ function at $\lambda_{0} \approx 1.4$. For when $\lambda>\lambda_{0}$ the 'cusp' structure begins to flatten, while for $\lambda<\lambda_{0}$ it sharpens. As has been shown, the effect of this change is quite significant; it introduces a discontinuity in the first derivative of the modeled mean behavior of $Q$. The result of which is a PDF that asymptotically approaches $\delta\left(Q-Q_{0}\right)$ instead of zero due to the reversal of the sign of the drift term at the discontinuity. The

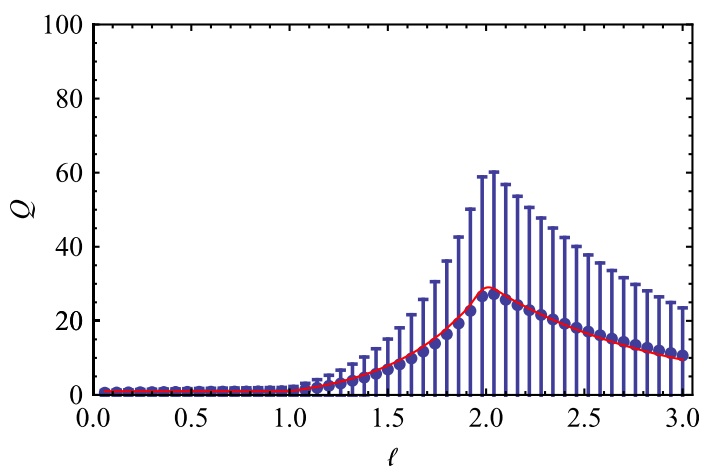

Fig. 9. The calculated mean $Q$ (circles) along with its standard deviation according to the OU-based PDF, Eq. (9). Solid line is the deterministic $Q$ as given by Eq. (1).

OU-based PDF's sensitivity to the structure parameter $\mu$ is also evident on Fig. 10, but this behavior is expected; being predicated by the form of Eq. (6).

The sensitivity of the OU-based PDF to the parameters of Eq. (1) is not, in contrast, easily discernable. This is precisely the reason why a rather complex function like Eq. (6) was chosen to characterize the mean behavior of $Q$ instead of the simpler form of Eq. (1); to characterize and analyze the various structures and their sensitivity to the model's structure parameters.

The main result of which is that the OU-based PDF appears particularly sensitive to any parameterization of the mean behavior of $Q$ with a discontinuous first derivative (through the scale parameter $\lambda$ in our model). The same PDF is only weakly dependent on the other structure parameters like asymmetry and tail thickness.

\subsection{Model sensitivity}

Assuming the proposed linear model is applicable (i.e., assumptions of an additive noise along with a persistent mean structure), here we try to answer the larger question as to how sensitive this linear model is to different representations of the
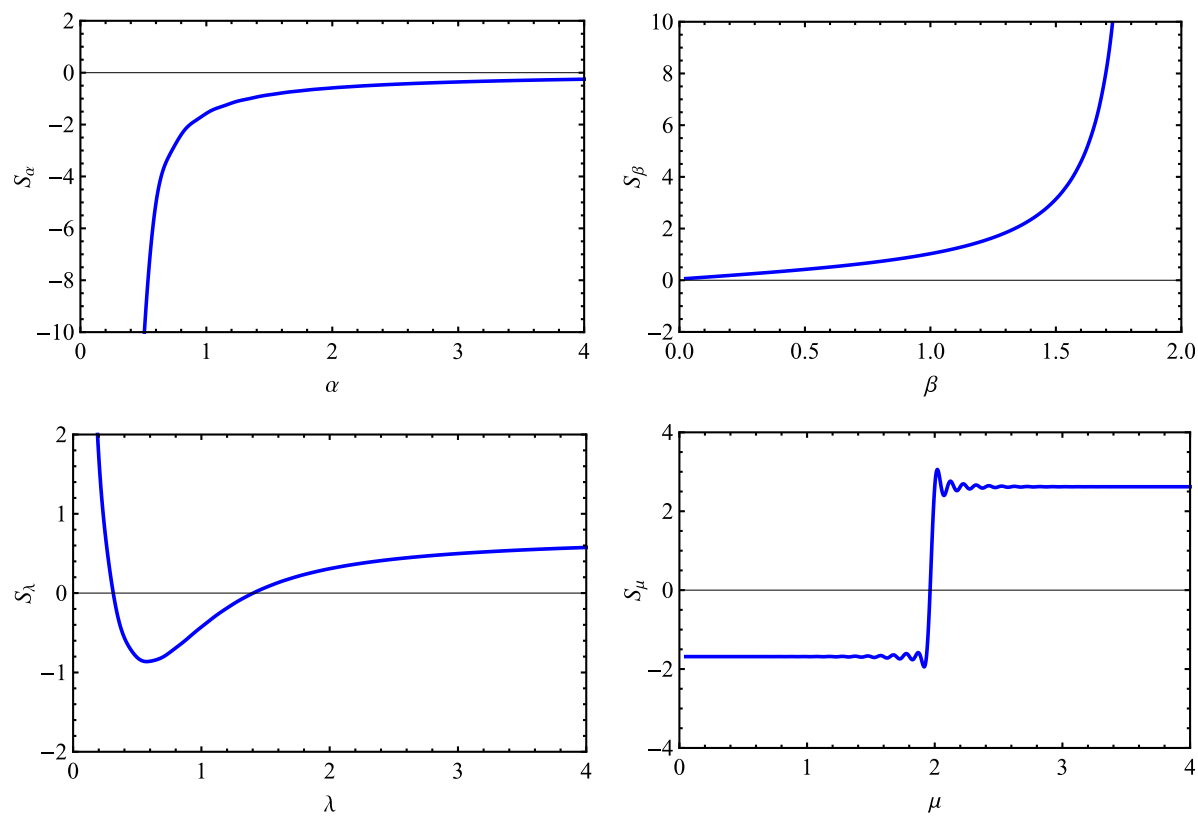

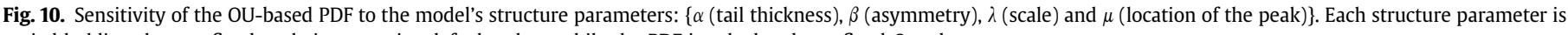
varied holding the rest fixed at their respective default values while the PDF is calculated at a fixed $Q$ and $\ell$. 
mean structure of $Q$. In the previous section, we have shown that the representation based on Eq. (1), without regard to the discontinuity in the first derivative, in a linear model context appears to give a rather similar solution (in the sense of the first two moments, at least) to that based on Eq. (6). We can address this question statistically (i.e., in the mean-square sense) by assuming two different linear models each given by Eq. (4), but whose drift and diffusion functions are given by Eq. (1) and Eq. (6), respectively, and then apply a filter like Kalman-Bucy filter (see, e.g., Oksendal, 2003). Assume then,

$d Q_{1}=C_{1}(\ell) Q_{1} d \ell+\sqrt{D_{1}(\ell)} d W_{1}$,

$d Q_{2}=C_{2}(\ell) Q_{2} d \ell+\sqrt{D_{2}(\ell)} d W_{2}$,

where $d W_{1}$ and $d W_{2}$ are uncorrelated. If we take "1" to refer to an accepted model for $Q$ and " 2 " to actual observations of $Q$ then, according to the Kalman-Bucy theorem, the best estimate of $Q Q^{o p}$, satisfies the following SDE:

$d Q^{o p}=\underbrace{\left(C_{1}(\ell)-\frac{C_{2}^{2}(\ell) S_{Q}(\ell)}{D_{2}(\ell)}\right)}_{\text {optimized drift }} Q^{o p} d \ell+\underbrace{\frac{C_{2}(\ell) S_{Q}(\ell)}{D_{2}(\ell)}}_{\text {optimized diffusion }} d W_{3}$,

while the mean-square error function, $S_{Q}(\ell)=E\left[\left(Q_{1}(\ell)-Q^{o p}(\ell)\right)^{2}\right]$, with $E[\cdots]$ being an expectation value, satisfies the following (Riccati-type) deterministic differential equation:

$\frac{d S_{Q}(\ell)}{d \ell}=-\frac{C_{2}^{2}(\ell)}{D_{2}(\ell)} S_{Q}^{2}(\ell)+2 C_{1}(\ell) S_{Q}(\ell)+D_{1}(\ell)$,

with the 'initial' condition $S_{Q}(\ell=0)=0$.

We define a sensitivity measure to the exact formulation of the model as,

$\delta Q(\ell)=\sqrt{S_{Q}^{12}(\ell)}-\sqrt{S_{Q}^{21}(\ell)}$,

where $S_{Q}^{12}$ is a solution to Eq. (16) assuming $Q_{1}$ is model and $Q_{2}$ is observed, and vice versa for $S_{Q}^{21}$. Fig. 11 is a plot of $\delta Q$ as a function of $\ell$ where it is evident that interchanging model with observations produces only a small error. Fig. 11 suggests that the linear stochastic model is rather insensitive to the precise functional form of the deterministic $Q$.

We can use the above filter to infer an "optimized" $Q$ insofar as noise is associated with any representation that embodies the various substructures of the deterministic $Q_{2}$ in the presence of the additive noise suggested by $Q_{1}$. The error in this particular $Q$ is

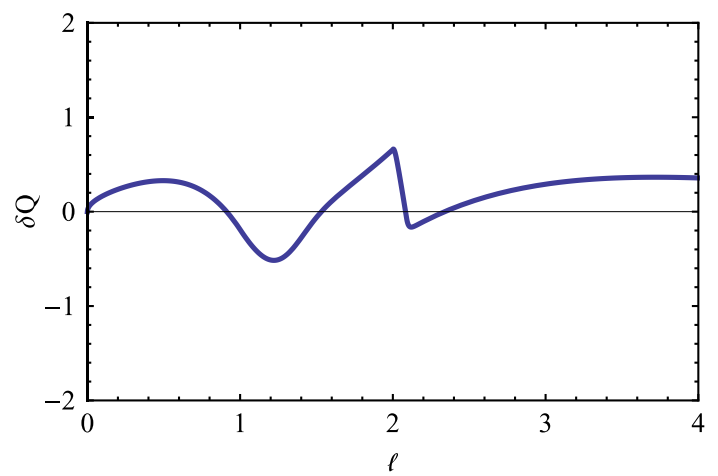

Fig. 11. Sensitivity to model measure, $\delta Q$ as a function of $\ell$ according to solutions of Eq. (16).

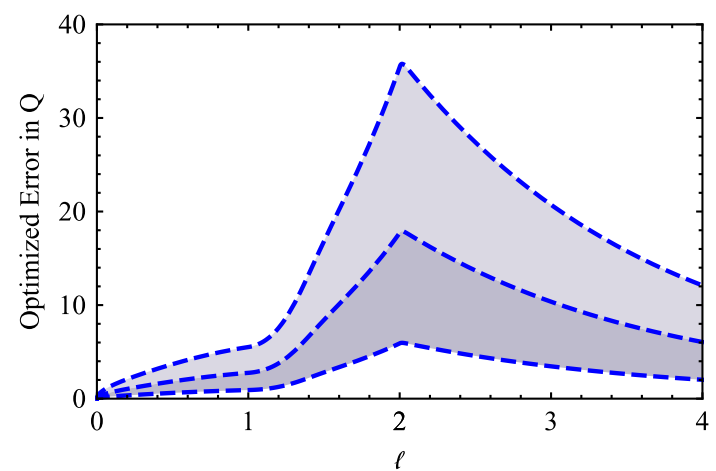

Fig. 12. Calculated error in the "optimized" $Q$ using Eq. (16). The error so depicted is bounded by 1,3 , and 6 root-mean-squares.

depicted on Fig. 12 where it is shown bounded by 1, 3 and 6 rootmean-squares, $\left(S_{Q}^{12}\right)^{1 / 2}$.

\section{Summary and conclusions}

In this work we have proposed and analyzed a simple stochastic dynamical model for a randomly fluctuating radiation quality factor, $Q$, based on the time-dependent Ornstein-Uhlenbeck process. In this proposed model, a normalized LET variable plays the role of the continuous time variable. The mean behavior of $Q$ as a function of LET is parameterized using the variance-gamma function so as to explicitly characterize the location of the peak, the asymmetry around the peak, the thickness of the asymptotic tail and the overall scale. The fluctuating part of $Q$ is assumed to be an additive noise term, as suggested by recent Monte-Carlo based simulations.

The proposed linear model's Fokker-Planck equation that corresponds to the model's stochastic equation, has an analytic solution that turns out to be a Gaussian with a linearly (in the logarithm of the variable LET) growing variance but with rather complex scaling properties. The derived probability density function's sensitivity to the structure parameters has been analyzed. The salient result of this analysis is that the Ornstein-Uhlenbeck based function appears to be quite sensitive to any parameterization of the mean behavior of $Q$ with a discontinuous first derivative in LET. In our particular parameterization, this is controlled by the scale parameter. The function appears to be only weakly dependent on the other structure parameters like asymmetry and tail thickness.

This discontinuity in the parameterized mean behavior of $Q$ makes its drift term in the proposed 'time'-dependent Ornstein-Uhlenbeck process discontinuous (through a sign change) at the peak. The result of which is a reflection of the solution at the peak: the density function asymptotically approaches zero in the absence of the discontinuity while it approaches the singular initial condition of $Q$ upon reflection at the discontinuity.

In addition to analyzing sensitivity to the structure parameters, using a Kalman-Bucy filter, we have shown the linear stochastic model to be rather insensitive to the precise functional form of the deterministic $Q$. Using this filter, we also have estimated the noise level in any linear model of $Q$ with the additive noise assumption. It may be possible to use this modeling variability to infer the true error or noise level in $Q$.

The cusp-like feature, seen both in standard representations of the deterministic $Q$ and in the stochastic model presented in this work, may, of course, be a spurious one necessitated by either modeling or be simply an artefact of the noise in the system. If empirically supported, however, it can, from a modeling 
perspective, be quite useful and insightful. For example, if the discontinuity is actually a true cusp-like structure (in the sense of elementary catastrophe theory, see, e.g., Thom, 1975; Stewart, 1982, 1983), it can, in principle, piece together a response of a change in the quality factor to collective changes in its 'control' variables, e.g., the scale and other structure parameters discussed earlier. This will allow, in a catastrophe-theoretic model of the quality factor, to capture dependencies among these and similar parameters that are otherwise difficult to model. Catastrophe theory based models have in the past been applied to various biological (Murray, 1993), biomedical (Seif, 1979), and behavioral (Casti, 1982) systems, but with a variable degree of success (Zeeman, 1977). Applications to radiobiology, like to the suggestive, cusp-like feature in the modeling of the radiation quality factor, may be potentially both powerful analytically as well as being computationally and numerically expedient.

\section{References}

Adams, J.H., Bhattacharya, M., Lin, Z.W., Pendleton, G., Watts, J.W., 2007. The ionizing radiation environment on the Moon. Adv. Space Res. 40, 33.

Alpen, E.L., 1998. Radiation Biophysics, 2nd. ed. Academic Press, San Diego.

Angelo Jr., J.A., Buden, D., 1985. Space Nuclear Power. Orbit Book, Florida.

Aziz, A., 2007. "Sensitivity analysis of the Q-factor", Marshall Academy 2007 Report pp. 11-16, NASA-MSFC, Alabama.

Barghouty, A.F., 2007. Optimization of Crew Shielding Requirement in ReactorPowered Lunar Surface Missions. NASA/TP-2007-215133. NASA STI, Maryland.

Barghouty, A.F., Thibeault, S.A., 2006. The Exploration Atmospheres Working Group's Report on Space Radiation Shielding Materials. NASA/TM-2006-214604. NASA STI, Maryland.

Casti, J., 1982. Toplogical Methods for Social and behavioral systems. Int. J. Gen. Syst. $8,187$.

Cucinotta, F.A., Kim, M.-H.Y., Ren, L., 2005. Managing Lunar and Mars Mission Radiation Risks Part I: Cancer Risks, Uncertainties, and Shielding Effectiveness. NASA/TP-2005-213164. NASA STI, Maryland.

Davis, J.M., Cataldo, R.L., Soeder, J.F., Monzo, M.A., Hakimzadeh, R., 2005. An Overview of Power Capability Requirements for Exploration Missions. NASA/TM2005-213600. NASA STI, Maryland.

Ferson, S., October 21-24, 1992. Using fuzzy Arihmetic in Monte-Carlo simulation is Fishery Populations. In: Proceedngs of the International Symposium on Managament Strategies for Exploited Fish Populations, pp. 595-608. Alaska.

Ferson, S., 1996. What Monte Carlo Methods Cannot do. Hum. Ecol. Risk Assess. 2, 990.
Ferson, S., Tucker, W.T., 2006. Sensitivty in Risk Analyses with Uncertain Numbers. Sandia Report no. SAND2006-2801. Sandia National Laboratories, New Mexico.

Gardiner, C.W., 1983. Handbook of Stochastic Methods for Physics, Chemistry, and the Natural Sciences. Springer, Berlin, Germany.

Hoffman, F.O., Hammonds, J.S., 1994. Propagation of uncertainty in risk assessments: the need to Distinguish between uncertainty due to lack of knowledge and uncertainty due to variability. Risk Anal. 14, 707.

International Commission on Radialogical Protection, ICRP Publication 26, 1977. Recommendations of the International Commission on Radiological Protection. Pergamon Press, New York.

International Commission on Radialogical Protection, ICRP Publication 60, 1991. Recommendations of the International Commission on Radiological Protection. Pergamon Press, New York.

International Commission on Radialogical Protection, ICRP Publication 92, 2003. Relative Biological Effectiveness (RBE), Quality Factor (Q), and Radiation Weighting Factor $\left(w_{R}\right)$. Elsevier Science Ltd., London, England.

Katz, R., Anderson, B., Homayoonfar, M., Sharma, S.C., 1971. Inactivation of cells by heavy ion bombardment. Radiat. Res. 47, 402.

Kerslake, T.W., 2005. Electric Power System Technology Options for Lunar Surface Missions. NASA/TM-2005-213629. NASA STI, Maryland.

Kudela, K., Storini, M., Hofer, M.Y., Belov, A., 2000. Cosmic rays in Relation to space Weather. Space Sci. Rev. 93, 153.

Lefebvre, M., 2000. Applied Stochastic Processes. Springer, Berlin, Germany.

Lo, C.F., Hui, C.H., 2006. Computing the first passage time density of timedependent Ornstein-Uhlenbeck process to a moving boundary. Appl. Math. Lett. 19, 1399.

Murray, J.D., 1993. Mathematical Biology. Springer, Berlin, Germany.

National Council on Radiation Protection's Report No. 98, (NCRP, Maryland, 1989) and Update on Guidance on Radiation Received in Space Activities, 1999. NCRP, Maryland.

Oksendal, B., 2003. Stochatic Differential Equations: An Introduction with Applications, sixth ed.. Springer, Berlin, Germany.

Rajkumar, T., Johnson, M., May 22-25, 2001. Population Viability analysis using fuzzy arithmetic. In: IFIP TC WG 5.11, 4th International Symposium on Environmental Software Systems (ISESS01), pp. 95-108. Banff, Alberta, Canada.

Seif, F.J., 1979. Cusp Bifurcation in Pituitary Thyrotropin Secretion. In: Guttinger, W., Eikemeier, H. (Eds.), Structural Stability in Physics. Springer, Berlin, Germany.

Stewart, I., 1982. Catastrophe theory in Physics. Rep. Prog. Phys. 45, 185.

Stewart, I., 1983. Elementary catastrophe theory. IEEE Trans. CAS 30, 98.

Thom, R., 1975. Structrual Stability and Morphogenesis. transl. by D.H. Fowler. Benjamin-Addison-Wesley, New York.

Wilson, J.W., Townsend, L.W., Schimmerling, W., Khandewal, G.S., Khan, F., Nealy, J.E., et al., 1991. Transport Methods and Interactions for Space Radiation. NASA/RP-1991-1257. NASA STI, Maryland.

Wilson, J.W., Miller, J., Konradi, A., Cucinotta, F.A. (Eds.), 1997. Shielding Strategies for Human Space Exploration. NASA Conf. Pub., vol. 3360. NASA STI, Maryland. Zeeman, E.C., 1977. Catastrophe Theory (Selected Papers). Addison-Weseley, Massachusetts. 